\title{
SOME DISCRETE APPROXIMATIONS TO A VARIATIONAL METHOD FOR IMAGE SEGMENTATION*
}

\author{
S.R. Kulkarni ${ }^{1,2}$ and S.K. Mitter ${ }^{1}$ \\ ${ }^{1}$ Laboratory for Information and Decision Systems, M.I.T. \\ Cambridge, MA 02139 \\ ${ }^{2}$ M.I.T. Lincoln Laboratory \\ Lexington, MA 02173
}

January 3, 1991

\begin{abstract}
Variational formulations have been proposed for a number of tasks in early vision. Discrete versions of these problems are closely related to Markov random field models and are typically used in implementing such methods. In particular, discrete and continuous versions for the problem of image segmentation have received considerable attention from both theoretical and algorithmic perspectives.

It has been previously pointed out that the usual discrete version of the segmentation problem does not properly approximate the continuous formulation in the sense that the discrete solutions may not converge to a solution of the continuous problem as the lattice spacing tends to zero. One method for modifying the discrete formulations to ensure such convergence has been previously discussed. Here we consider two other partially discrete formulations which also satisfy desirable convergence properties in the continuum limit, and we discuss some general ideas about digitized versions of the variational formulation of the segmentation problem.
\end{abstract}

*This work was supported by the U.S. Army Research Office under Contract DAAL03-86-K-0171, the Air Force Office of Scientific Research under grant AFOSR 89-0276 and by the Department of the Navy under Air Force Contract F19628-90-C-0002. 


\section{Introduction}

Recently, many porblems in early vision have been formulated using variational approaches (e.g., see [18]). These variational methods are appealing for a number of reasons. The terms in the cost function are intuitively plausible and correspond in a natural way to constraints generally expected to be present in the environment (for example certain invariants and/or smoothness). Also, these methods provide a unifying approach to the wide variety of early vision tasks, and in fact suggest ways in which various early vision modules might be fused. Finally, it is possible to analyze certain problems (such as for segmentation) to obtain general properties of the solutions provided by these variational techniques.

In practice, these variational methods are generally implemented using finite-difference-like approximations with discrete versions of the cost function defined on digitized domains. Interestingly, the resulting discrete problems are closely related to Markov random field (MRF) models, which are conceptually and computationally appealing. In fact, for many problems the MRF formulation is the starting point from which a variational principle on a continuous domain is deduced if necessary.

A continuous formulation is useful for a number of reasons. For example, it may be easier to impose or identify certain constraints such as invariance under arbitrary rotations and translations. Also, analytical techniques can be more readily applied to derive properties of the continuous formulation. However, since analytic solutions are not available, the problem must eventually be digitized to obtain numerical solutions. The discrete problem has the advantages of being more directly amenable to computer implementations, particularly with parallel algorithms or hardware. Also, as mentioned above, the probabilistic interpretation in terms of MRF's is conceptually appealing.

A natural question is whether these discrete formulations are in fact approximations of the continuous formulations in the sense that solutions to the discrete problems are close to solutions of the continuous problem as the lattice spacing tends to zero. This question is important if one wants to guarantee that the advantages of the continuous formulations are retained, at least approximately, by solving the discrete problem. In fact, the main criteria for considering a discrete formulation to be an "approximation" to a continuous problem should not be whether the cost functions are approximations of one another in the usual sense, but rather whether the solutions provided by the two problems (i.e., the minimizers of the cost functions) are approximations of one another.

Here we consider these questions of suitable discrete approximations for a particular formulation for image restoration and segmentation. Various discrete formulations in terms of MRF's were studied by Geman and Geman [12], Marroquin [17] and others. A variational approach to the problem was proposed by Mumford and Shah in [22] (see also Blake and Zisserman [4, 5]). Below, we first describe the variational method for the problem of reconstructing and segmenting an image degraded by noise, and then the usual discrete approximation proposed for this problem.

The variational method involves minimizing a cost functional over a space of boundaries with suitably smooth functions within the boundaries. Specifically, if $g$ represents the observed image 
defined on $\Omega \subset \mathbf{R}^{2}$, then a reconstructed image $f$ and its associated edges $\Gamma$ are found by minimizing

$$
E(f, \Gamma)=c_{1} \iint_{\Omega}(f-g)^{2} d x d y+c_{2} \iint_{\Omega \backslash \Gamma}\|\nabla f\|^{2} d x d y+c_{3} L(\Gamma)
$$

where $c_{1}, c_{2}, c_{3}$ are constants and $L(\Gamma)$ denotes the length of $\Gamma$. An interesting special case of this problem is obtained if $f$ is restricted to be constant within connected components of $\Omega \backslash \Gamma$. In this case, the optimal value of $f$ on a connected component of $\Omega \backslash \Gamma$ is simply the mean of $g$ over the connected component. Hence, the solution depends only on $\Gamma$ and is obtained by minimizing

$$
E(\Gamma)=c_{1} \sum_{i=1}^{k} \iint_{\Omega_{i}}\left(g-\bar{g}_{i}\right)^{2} d x d y+c_{3} L(\Gamma)
$$

where $\Omega_{1}, \ldots, \Omega_{k}$ are the connected components of $\Omega \backslash \Gamma$, and $\bar{g}_{i}$ is the mean of $g$ over $\Omega_{i}$.

In the usual discrete versions of this problem [5, 22] (see also the MRF formulations of [12, 17]), the original image $g$ is defined on a subset of the lattice $\frac{1}{n} \mathbf{Z}^{2}$ with lattice spacing $\frac{1}{n}$. The reconstructed image $f$ is defined on the same lattice, while the boundary $\Gamma$ consists of a subset of line segments joining neighboring points of the dual lattice. For the discrete problem, $f$ and $\Gamma$ are found by minimizing

$$
E(f, \Gamma)=c_{1} \sum_{i \in \Omega} \frac{1}{n^{2}}\left(f_{i}-g_{i}\right)^{2}+c_{2} \sum_{\substack{i, i^{\prime} \in \Omega \\ \text { adjacent } \\ i i^{\prime} \cap \Gamma=\emptyset}}\left(f_{i}-f_{i^{\prime}}\right)^{2}+c_{3} L(\Gamma)
$$

As discussed in $[14,15]$, it seems that the discrete problem given above does not properly approximate the continuous problem in the sense that solutions to (3) may not necessarily converge to a solution of (1) as $n \rightarrow \infty$. An alternate discrete formulation was proposed in [14, 15] which involved modifications to both the cost functional and the dicretization procedure. The use of Minkowski content as the penalty term for the boundaries was considered, and the discrete boundaries were taken to be a union of closed lattice squares rather than a union of line segments. It was shown that, under certain conditions, solutions to this discrete formulation converge in the continuum limit to solutions of the continuous problem.

In this paper we discuss some other possibilities for discrete formulations and prove similar convergence results in these cases. As in $[14,15]$, for the discretization procedure we consider only digitizing the boundary. The observed and reconstructed images are still defined on continuous domains. The alternate formulations are discussed in Sections 3 and 4, after some preliminary definitions and results are given in Section 2.

\section{Background and Previous Work}

In this section we introduce a variety of notions useful in dealing with the 'boundaries' or 'edges' of an image. The 'image' is usually a real valued function defined on a bounded open set $\Omega \subset \mathbf{R}^{2}$, although some of the results consider the more general case of $\Omega \subset \mathbf{R}^{n}$. A boundary generally refers to a closed subset of $\bar{\Omega}$. However, sometimes the boundary may be restricted to have certain 
additional properties such as having a finite number of connected components. A topology on the space of boundaries is required for the notion of convergence, and a measure of the 'cost' of a boundary is required for the variational problem.

For $A \subset \mathbf{R}^{n}$, the $\delta$-neighborhood of $A$ will be denoted by $A^{(\delta)}$ and is defined as

$$
A^{(\delta)}=\left\{x \in \mathbf{R}^{n}: \inf _{y \in A}|x-y|<\delta\right\}
$$

The notion of distance between boundaries which we will use is the Hausdorff metric $d_{H}(\cdot, \cdot)$ defined as

$$
d_{H}\left(A_{1}, A_{2}\right)=\inf \left\{\rho: A_{1} \subset A_{2}^{(\rho)} \text { and } A_{2} \subset A_{1}^{(\rho)}\right\}
$$

It is elementary to show that $d_{H}(\cdot, \cdot)$ is in fact a metric on the space of all non-empty compact subsets of $\mathbf{R}^{n}$. An important and well known property of this metric is that it induces a topology which makes the space of boundaries compact.

For the 'cost' of a boundary, the usual notion of length cannot be applied to highly irregular boundaries. Hence a measure on the space of boundaries which generalizes the usual notion of length is desired. A variety of such measures for subsets of $\mathbf{R}^{n}$ have been investigated. (e.g., see [10]). Perhaps the most widely used and studied are Hausdorff measures $[9,10,26]$.

For a non-empty subset $A$ of $\mathbf{R}^{n}$, the diameter of $A$ is defined by $|A|=\sup \{|x-y|: x, y \in A\}$. Let

$$
\omega_{s}=\frac{\Gamma\left(\frac{1}{2}\right)^{s}}{\Gamma\left(\frac{s}{2}+1\right)}
$$

where $\Gamma(\cdot)$ is the usual Gamma function. For integer values of $s, \omega_{s}$ is the volume of the unit ball in $\mathbf{R}^{s}$. For $s>0$ and $\delta>0$ define

$$
\mathcal{H}_{\delta}^{s}(A)=2^{-s} \omega_{s} \inf \left\{\sum_{i=1}^{\infty}\left|U_{i}\right|^{s}: A \subset \bigcup_{i=1}^{\infty} U_{i},\left|U_{i}\right| \leq \delta\right\}
$$

The Hausdorff s-dimensional measure of $A$ is then given by

$$
\mathcal{H}^{s}(A)=\lim _{\delta \rightarrow 0} \mathcal{H}_{\delta}^{s}(A)=\sup _{\delta>0} \mathcal{H}_{\delta}^{s}(A)
$$

Note that the factor $2^{-s} \omega_{s}$ in the definition of $H_{\delta}^{s}(\cdot)$ is included for proper normalization. With this definition, for integer values of $s$, Hausdorff measure gives the desired value on sets where the usual notions of length, area, and volume apply.

Many properties of Hausdorff measure can be found in $[9,10,26]$. The following definitions are required to state several useful properties. A curve $\Gamma \subset \mathbf{R}^{n}$ is the image of a continuous injection $\psi:[0,1] \rightarrow \mathbf{R}^{n}$. The length of a curve $\Gamma$ is defined as

$$
L(\Gamma)=\sup \left\{\sum_{i=1}^{m}\left|\psi\left(t_{i}\right)-\psi\left(t_{i-1}\right)\right|: 0=t_{0}<t_{1}<\cdots<t_{m}=1\right\}
$$

and $\Gamma$ is said to be rectifiable if $L(\Gamma)<\infty$. Finally, a compact connected set is called a continuum. Some useful properties of Hausdorff measure are that the 1-dimensional Hausdorff measure of a curve is equivalent to the length of the curve, and that $\mathcal{H}^{1}$-measure is lower-semicontinuous on the 
set of connected boundaries with respect to the Hausdorff metric - i.e., if $\left\{\Gamma_{n}\right\}$ is a sequence of continuua in $\mathbf{R}^{n}$ that converges (in Hausdorff metric) to a compact set $\Gamma$, then $\Gamma$ is a continuum and $\mathcal{H}^{1}(\Gamma) \leq \liminf _{n \rightarrow \infty} \mathcal{H}^{1}\left(\Gamma_{n}\right)$.

Richardson $[24,25]$ extended the lower semicontinuity result to a cost term for boundaries which depends on the number of connected components. Specifically, define $\nu(\Gamma)=\mathcal{H}^{1}(\Gamma)+F(\#(\Gamma))$ where $\#(\Gamma)$ denotes the number of connected components of $\Gamma$, and $F$ is any non-decreasing function such that $\lim _{n \rightarrow \infty} F(n)=\infty$. Then $\#(\cdot)$ and $\nu(\cdot)$ are lower-semicontinuous on the space of boundaries with respect to the Hausdorff metric. This result was used in [24] to prove an existence theorem for the variational problems of interest. The essential properties required are the compactness of the space of boundaries and the lower-semicontinuity of the cost functional. However, with the discrete approximations suggested in $[5,22]$ and elsewhere, the cost for the boundaries can be strictly lower-semicontinuous with the topology induced by the Hausdorff metric. It is for this reason that discrete solutions may fail to converge in the continuum limit to a solution of the continuous problem.

It is possible to resolve this problem by modifying the cost functional and/or the discretization process. In $[14,15]$ an alternate notion for the cost of the boundaries and a modified discretization process were considered. Specifically, Minkowski content [10] was used as the cost term for the boundaries, and the discrete boundaries were represented by a union of closed lattice squares (as described in the next section) rather than the union of line segments separating lattice sites as is usually done. Several properties of Minkowski content were shown in $[14,15]$ which were used to show convergence properties of partially discrete versions of (1). Other approaches to digitizing the problem have been considered and implemented (e.g., in [25, 11]), although proofs of convergence of these methods are lacking (see Section 5 for more discussion). In the next two sections we consider two new methods for digitizing (1) for which we prove convergence results. Although, the methods are difficult to implement, the proofs of convergence are substantially simpler than that using Minkowski content. Furthermore, consideration of several alternative discretization methods suggests some general properties of discrete versions of the continuous problem as discussed in Section 5 .

\section{Alternate Cost for Discrete Boundaries}

In this section we propose a discrete version of (1) which possesses the desirable convergence property in the continuum limit. As in the discretized version using Minkowski content [14, 15], the discrete boundaries consist of unions of discrete closed lattice squares. However, we assign a different cost (measure of length) to the discrete boundaries. It is shown that this alternate measure of length satisfies a convergence property from which convergence of solutions to the variational problem follows.

For integers $i, j$ let

$$
S_{i j}=\left\{\left(x_{1}, x_{2}\right) \in \mathbf{R}^{2}: i \leq x_{1} \leq i+1, j \leq x_{2} \leq j+1\right\}
$$

$S_{i j}$ is the closed unit square whose lower left corner is at $(i, j)$. Then $\frac{1}{n} S_{i j}$ is a square with side $\frac{1}{n}$ whose lower left corner is at $(i / n, j / n)$. For the discrete problem with lattice spacing $\frac{1}{n}$, the boundaries consist of a union of the $\frac{1}{n} S_{i j}-$ i.e., a union of closed lattice squares of $\frac{1}{n} \mathbf{Z}^{2}$. The 
discrete version with spacing $\frac{1}{n}$ of a boundary $\Gamma \subset \mathbf{R}^{2}$ is given by $P_{n}(\Gamma)$ where $P_{n}(\cdot)$ maps $\Gamma$ to the subset of $\mathbf{R}^{2}$ consisting of the union of the $\frac{1}{n} S_{i j}$ that $\Gamma$ intersects:

$$
P_{n}(\Gamma)=\bigcup_{\substack{i, j w i t h \\ \frac{1}{n} S_{i j} \cap \Gamma \neq \emptyset}} S_{i j}
$$

We can now define an alternate cost for discrete boundaries (or rather discrete approximations to length for any boundary). Let $\Gamma \subset \mathbf{R}^{2}$. First, suppose that $P_{n}(\Gamma)$ (the discrete version on the lattice with spacing $1 / n)$ is connected. In this case, define $\mathcal{L}_{n}(\Gamma)$ by

$$
\mathcal{L}_{n}(\Gamma)=\inf \left\{\mathcal{H}^{1}(\Lambda): \Lambda \text { connected and } P_{n}(\Lambda)=P_{n}(\Gamma)\right\}
$$

In the general case, define $\mathcal{L}_{n}(\Gamma)$ by

$$
\mathcal{L}_{n}(\Gamma)=\sum_{i} \mathcal{L}_{n}\left(G_{i}\right)
$$

where the $G_{i}$ are the connected components of $P_{n}(\Gamma)$.

The discrete measure of length assigned to a connected discrete boundary $P_{n}(\Gamma)$ is the minimum length of a connected curve that gives rise to the same discrete boundary. Related measures of length for discrete boundaries have been studied in $[13,20,21]$. Note that for bounded $\Gamma$, the inf could actually be replaced by min since the infimum is achieved. This follows from the compactness result of compact sets under the Hausdorff metric and the lower semicontinuity of Hausdorff measure. The sum in the extension to arbitrary boundaries is well defined since any discrete boundary has at most a countable number of connected components. In fact, a bounded discrete boundary has a finite number of connected components. Two boundaries that give rise to the same discrete boundary have the same discrete measure of length.

Using this discrete measure of length, we can define a partially discrete version of the original variational problem (1) with lattice spacing $\frac{1}{n}$. The boundaries are reconstructed only to within their $\frac{1}{n}$ discrete versions, but the observed and reconstructed images are still defined on continuous domains. Specifically, given an observed image $g \in L^{\infty}(\Omega)$ we reconstruct a discrete boundary $P_{n}(\Gamma)$ and an image $f$ on $\Omega \backslash P_{n}(\Gamma)$ by minimizing

$$
E_{n}\left(f, P_{n}(\Gamma)\right)=c_{1} \iint_{\Omega \backslash P_{n}(\Gamma)}(f-g)^{2} d x d y+c_{2} \iint_{\Omega \backslash P_{n}(\Gamma)}\|\nabla f\|^{2} d x d y+c_{3} \mathcal{L}_{n}\left(P_{n}(\Gamma)\right)
$$

We now discuss some properties of the discrete measure of length and the associated discrete variational problem.

Theorem 1 For every fixed $n<\infty$, a minimizing solution $\left(f^{*}, P_{n}\left(\Gamma^{*}\right)\right)$ for $E_{n}(\cdot, \cdot)$ exists.

Proof: For a fixed boundary, the minimization of $E_{n}$ is a standard variational problem for which a solution exists. Since $\Omega$ is assumed to be bounded, there are only a finite number of distinct discrete boundaries $P_{n}(\Gamma)$, and so the result follows.

A common way to prove existence to variational problems is to show a compactness property and lower-semicontinuity of the cost function. Due to the finite number of possible discrete boundaries, 
compactness and lower-semicontinuity over the set of discrete boundaries are immediate. However, $\mathcal{L}_{n}(\cdot)$ is not lower-semicontinuous over all $\Gamma$. For a simple example, take $n=1$, and let $\Gamma_{k}$ be the straight line joining $\left(\frac{1}{2}, \frac{1}{2}\right)$ and $\left(2-\frac{1}{k}, \frac{1}{2}\right)$. Then $P_{1}\left(\Gamma_{k}\right)=S_{00} \cup S_{10}$ so that $\mathcal{L}_{1}\left(\Gamma_{k}\right)=0$. Also, the $\Gamma_{k}$ converge to the straight line $\Gamma$ joining $\left(\frac{1}{2}, \frac{1}{2}\right)$ and $\left(2, \frac{1}{2}\right)$, so that $P_{1}(\Gamma)=S_{00} \cup S_{10} \cup S_{20}$. Therefore, $\mathcal{L}_{1}(\Gamma)=1>\lim \inf _{k \rightarrow \infty} \mathcal{L}_{1}\left(\Gamma_{k}\right)=0$.

The following result shows that for a large class of boundaries the discrete measures of length converge to the usual notion of length as $n \rightarrow \infty$.

Theorem 2 If $\Gamma$ is a compact set with a finite number of connected components then $\lim _{n \rightarrow \infty} \mathcal{L}_{n}(\Gamma)=\mathcal{H}^{1}(\Gamma)$.

Proof: If $G_{1}, \ldots, G_{m}$ are the connected components of $\Gamma$, then for sufficiently large $n$ the $P_{n}\left(G_{i}\right)$ are disjoint for $i=1, \ldots, m$. In that case, $\mathcal{L}_{n}(\Gamma)=\sum_{i=1}^{m} \mathcal{L}_{n}\left(G_{i}\right)$. Also, $\mathcal{H}^{1}(\Gamma)=\sum_{i=1}^{m} \mathcal{H}^{1}\left(G_{i}\right)$. The result will follow if it can be shown for each connected component. Therefore, suppose that $\Gamma$ is connected.

By the definition of $\mathcal{L}_{n}(\Gamma)$, we have $\mathcal{L}_{n}(\Gamma) \leq \mathcal{H}^{1}(\Gamma)$ so that $\lim \sup _{n \rightarrow \infty} \mathcal{L}_{n}(\Gamma) \leq \mathcal{H}^{1}(\Gamma)$. On the other hand, for each $n$, there exists a compact and connected $\Lambda_{n}$ with $P_{n}\left(\Lambda_{n}\right)=P_{n}(\Gamma)$ and $\mathcal{H}^{1}\left(\Lambda_{n}\right)=\mathcal{L}_{n}(\Gamma)$. Since $P_{n}\left(\Lambda_{n}\right)=P_{n}(\Gamma), \Lambda_{n} \rightarrow \Gamma$ in Hausdorff metric as $n \rightarrow \infty$. By the lower-semicontinuity of $\mathcal{H}^{1}$ (see [9], Theorem 3.18) we have $\mathcal{H}^{1}(\Gamma) \leq \lim \inf _{n \rightarrow \infty} \mathcal{H}^{1}\left(\Lambda_{n}\right)=$ $\lim \inf _{n \rightarrow \infty} \mathcal{L}_{n}(\Gamma)$. Thus, $\lim _{n \rightarrow \infty} \mathcal{L}_{n}(\Gamma)$ exists and equals $\mathcal{H}^{1}(\Gamma)$.

The following theorem shows that the discrete measures of length converge in another useful way to Hausdorff measure.

Theorem $3 \mathcal{L}_{n}(\cdot)$ is $\Gamma$-convergent to $\mathcal{H}^{1}(\cdot)$ on the space of compact subsets of $\mathbf{R}^{2}$ with a bounded number of connected components and with the topology induced by the Hausdorff metric. I.e., let $\Gamma \subset \mathbf{R}^{2}$ be compact with $\#(\Gamma) \leq M<\infty$. Then the following two conditions hold:

(i) For every sequence of compact sets $\Gamma_{n} \subset \mathbf{R}^{2}$ with $\Gamma_{n} \rightarrow \Gamma$ in Hausdorff metric and \# $\left(\Gamma_{n}\right) \leq$ $M \forall n$ we have

$$
\mathcal{H}^{1}(\Gamma) \leq \liminf _{n \rightarrow \infty} \mathcal{L}_{n}\left(\Gamma_{n}\right)
$$

(ii) There exists a sequence of compact sets $\Gamma_{n} \subset \mathbf{R}^{2}$ with $\Gamma_{n} \rightarrow \Gamma$ in Hausdorff metric and $\#\left(\Gamma_{n}\right) \leq M \forall n$ such that

$$
\mathcal{H}^{1}(\Gamma) \geq \underset{n \rightarrow \infty}{\lim \sup } \mathcal{L}_{n}\left(\Gamma_{n}\right)
$$

Proof: To show (i), let $\Gamma_{n}$ be any sequence of compact sets with $\#\left(\Gamma_{n}\right) \leq M$ and $\Gamma_{n} \rightarrow \Gamma$ in Hausdorff metric. Then $\#\left(P_{n}\left(\Gamma_{n}\right)\right) \leq \#\left(\Gamma_{n}\right) \leq M$. Therefore, for each $n$ there exists $\Lambda_{n}$ with $\#\left(\Lambda_{n}\right)=\#\left(P_{n}\left(\Gamma_{n}\right)\right) \leq M, P_{n}\left(\Lambda_{n}\right)=P_{n}\left(\Gamma_{n}\right)$, and $\mathcal{H}^{1}\left(\Lambda_{n}\right)=\mathcal{L}_{n}\left(\Gamma_{n}\right)$. Since $\Gamma_{n} \rightarrow \Gamma$, we have $P_{n}\left(\Gamma_{n}\right) \rightarrow \Gamma$ and also since $P_{n}\left(\Lambda_{n}\right)=P_{n}\left(\Gamma_{n}\right)$ we have $\Lambda_{n} \rightarrow \Gamma$. Hence, by the lower-semicontinuity of Hausdorff measure for a bounded number of connected components (as mentioned in Section 2 - see $[24,25])$, we get $\mathcal{H}^{1}(\Gamma) \leq \liminf _{n \rightarrow \infty} \mathcal{H}^{1}\left(\Lambda_{n}\right)=\liminf _{n \rightarrow \infty} \mathcal{L}_{n}\left(\Gamma_{n}\right)$. 
To show (ii), simply take $\Gamma_{n}=\Gamma$ for all $n$. Then by the definition of $\mathcal{L}_{n}(\Gamma)$, we have $\mathcal{H}^{1}(\Gamma) \geq$ $\mathcal{L}_{n}(G)=\mathcal{L}_{n}\left(\Gamma_{n}\right)$ for all $n$ and so $\mathcal{H}^{1}(\Gamma) \geq \lim \sup _{n \rightarrow \infty} \mathcal{L}_{n}\left(\Gamma_{n}\right)$.

The $\Gamma$-convergence property shown above is sufficient to show convergence of solutions to the discrete problem (4) as the lattice spacing $\frac{1}{n}$ goes to zero if the number of connected components of the admissible boundaries is uniformly bounded. I.e., following [24], we let the cost term for the boundaries be

$$
\nu_{n}(\Gamma)=\mathcal{L}_{n}(\Gamma)+F(\#(\Gamma))
$$

where $F(k)=0$ for $k \leq M<\infty$ and $F(k)=\infty$ for $k>M$. Let $E_{n}^{M}$ denote the cost functional with the above boundary term, and let $E^{M}$ denote the cost functional whose boundary term is

$$
\nu(\Gamma)=\mathcal{H}^{1}(\Gamma)+F(\#(\Gamma))
$$

For these variational problems, we have the following convergence result, which essentially follows from the previous result on $\Gamma$-convergence.

Theorem 4 Let $\left(f_{n}^{*}, \Gamma_{n}^{*}\right)$ denote a minimizing pair for $E_{n}^{M}$. Then there exists a subsequence (still denoted $\left.\left(f_{n}^{*}, \Gamma_{n}^{*}\right)\right)$ and a pair $(f, \Gamma)$ such that $\left(f_{n}^{*}, \Gamma_{n}^{*}\right) \rightarrow(f, \Gamma)$ and $(f, \Gamma)$ minimizes $E^{M}$.

Proof: The existence of a pair $(f, \Gamma)$ with $\left(f_{n}^{*}, \Gamma_{n}^{*}\right) \rightarrow(f, \Gamma)$ follows from Lemma 3 of [24]. The fact that $(f, \Gamma)$ minimizes $E^{M}$ follows from the $\Gamma$-convergence of $\mathcal{L}_{n}$ to $\mathcal{H}^{1}$ (ahown above) which implies the $\Gamma$-convergence of $E_{n}^{M}$ to $E^{M}$.

\section{Segmentation with Piecewise Linear Boundaries}

In this section we formulate a modified version of the original variational problem (1) which eliminates the problems associated with computing the length of irregular curves. The modification consists of requiring the boundaries $\Gamma$ to consist of a union of a bounded number of straight line segments. We propose a partially discrete version of the modified problem and show a convergence result for the solutions to the discrete problems.

Let $\mathcal{S}^{M}=\mathcal{S}^{M}(\Omega)$ be the set of all compact subsets of $\Omega$ that are the union of $M<\infty$ or fewer connected line segments contained in $\Omega$. Consider the problem of minimizing (1) subject to the constraint that $\Gamma \in \mathcal{S}^{M}$. Alternatively, we can consider this problem as one of minimizing the cost function $E_{\mathcal{S}^{M}}$ whose boundary term is $\mathcal{H}^{1}(\Gamma)$ for $\Gamma \in \mathcal{S}^{M}$ and infinite otherwise.

Theorem 5 For every fixed $M<\infty$ and $\Omega$ a bounded region of $\mathbf{R}^{2}, \mathcal{S}^{M}(\Omega)$ is compact with respect to the Hausdorff metric. I.e., for every infinte sequence $\Gamma_{n} \in \mathcal{S}^{M}(\Omega)$ there is a subsequence, still denoted $\Gamma_{n}$, and $\Gamma \in \mathcal{S}^{M}(\Omega)$ such that $\Gamma_{n} \rightarrow \Gamma$ in Hausdorff metric. 
Proof: First, each $\Gamma_{n}$ is a compact subset of $\Omega$. As mentioned in Section 2, the set of compact subsets of $\Omega$ is compact with respect to the Hausdorff metric (e.g., see [9], Theorem 3.16). Hence, there is a subsequence, which we still denote $\Gamma_{n}$, and a compact set $\Gamma \subset \Omega$ such that $\Gamma_{n} \rightarrow \Gamma$ in Hausdorff metric. We need only show that $\Gamma \in \mathcal{S}^{M}(\Omega)$, i.e., that $\Gamma$ is the union of at most $M$ line segments.

Note that by a line segement we include the possibility of a single point or the empty set. It is straightforward to show the result for $M=1$. I.e., given a sequence of single line segments any convergent subequence converges to a single line segment. Now, each $\Gamma_{n}$ is the union of at most $M$ line segments so that $\Gamma_{n}=\cup_{i=1}^{M} A_{n, i}$ where each $A_{n, i}$ is a line segment (possibly a point or the empty set).

We can extract a subsequence ( still indexed by $n$ ) so that $A_{n, 1} \rightarrow A_{1}$ where $A_{1}$ is a line segment (again, possibly a point or the empty set). Similarly, we can extract sub-subsequences $M-1$ more times ( still indexed by $n$ ) so that for each $i=1, \ldots, M, A_{n, i} \rightarrow A_{i}$ where each $A_{i}$ is a line segment.

Therefore, for the final sub-subsequence, $\Gamma_{n}=\cup_{i=1}^{M} A_{n, i}$ and for each $i=1, \ldots, m$ we have $A_{n . i} \rightarrow A_{i}$ where $A_{i}$ is a line segment. Hence, $\Gamma_{n} \rightarrow \Gamma=\cup_{i=1}^{M} A_{i}$ and so $\Gamma \in \mathcal{S}^{M}(\Omega)$.

Theorem 6 A minimizing solution $\left(f^{*}, \Gamma^{*}\right)$ for $E_{\mathcal{S}_{M}}(\cdot, \cdot)$ exists.

Proof: This follows from the compactness of $\mathcal{S}^{M}$ shown above and the lower-semicontinuity of the cost function (e.g., see $[24,25])$.

Now we consider partially discrete versions of the problem $E_{\mathcal{S}^{M}}$. As before, only the boundary is discretized. For this problem we consider a different form of discrete representation for the boundaries, taking advantage of the fact that the boundaries are piecewise linear. Specifically, we consider the set of lattice points in $1 / n \mathbf{Z}^{2}$, and require the endpoints of each line segment in the boundary to lie on these lattice points. Let $\mathcal{S}^{M, n}=\mathcal{S}^{M, n}(\Omega)$ denote the collection of all sets consisting of $M$ straight line segments whose endpoints lie in $\frac{1}{n} \mathbf{Z}^{2} \cap P_{n}(\Omega)$. To obtain a convergence result we will consider the boundary to be a dilation of the linear segments where the amount of dilation is related to the lattice spacing. For the discrete problem with lattice spacing $1 / n$, the cost function is minimized over boundaries of the form $\Gamma^{\left(\delta_{n}\right)}$ where $\delta_{n}>\sqrt{2} / n$ with $\delta_{n} \rightarrow 0$ as $n \rightarrow \infty$ and $\Gamma \in \mathcal{S}^{M, n}(\Omega)$. The reconstructed image is then defined only on $\Omega \backslash \Gamma^{\left(\delta_{n}\right)}$. However, the cost of the dilated boundary $\Gamma^{\left(\delta_{n}\right)}$ is taken to be simply the the total length of the straight line segments comprising $\Gamma$. Let $E_{\mathcal{S} M, n}$ denote the discrete problem with lattice spacing $1 / n$. For these discrete problems, we have the following convergence result.

Theorem 7 Let $\left(f_{n}^{*}, \Gamma_{n}^{*}\right)$ denote a minimizing pair for $E_{\mathcal{S} M, n}$. Then there exists a subsequence (still denoted $\left.\left(f_{n}^{*}, \Gamma_{n}^{*}\right)\right)$ and a pair $(f, \Gamma)$ such that $\left(f_{n}^{*}, \Gamma_{n}^{*}\right) \rightarrow(f, \Gamma)$ and $(f, \Gamma)$ minimizes $E_{\mathcal{S} M}$.

Proof: The existence of a pair $(f, \Gamma)$ with $\left(f_{n}^{*}, \Gamma_{n}^{*}\right) \rightarrow(f, \Gamma)$ follows from the compactness of $\mathcal{S}^{M}$ and Lemma 3 of [24]. We only need to show that $(f, \Gamma)$ minimizes $E_{\mathcal{S}}$. 
Let $\left(f^{*}, \Gamma^{*}\right)$ minimize $E_{\mathcal{S}^{M}}$. For each $n$, let $\Lambda_{n}$ be obtained from $\Gamma^{*}$ by taking the best approximation to $\Gamma^{*}$ using line segments whose endpoints lie in $\frac{1}{n} \mathbf{Z}^{2} \cap P_{n}(\Omega)$, i.e. the best boundary which can be used in the discrete problem with lattice spacing $\frac{1}{n}$. This will be obtained by taking the line segments whose endpoints lie on the lattice sites closest to the endpoints of $\Gamma^{*}$. Note that certainly $\Lambda_{n}^{\left(\delta_{n}\right)} \supset \Gamma^{*}$ since $\delta_{n}>\sqrt{2} / n$ and with lattice spacing $\frac{1}{n}$ there is always a lattice point within $\sqrt{2} / n$ of any point in $\Omega$. Let $h_{n}$ be the restriction of $f^{*}$ to $\Omega \backslash \Lambda_{n}^{\left(\delta_{n}\right)}$. Then, we have

$$
\begin{aligned}
E_{\mathcal{S} M}(f, \Gamma) & \leq \liminf _{n \rightarrow \infty} E_{\mathcal{S} M, n}\left(f_{n}^{*}, \Gamma_{n}^{*}\right) \leq \liminf _{n \rightarrow \infty} E_{\mathcal{S} M, n}\left(h_{n}, \Lambda_{n}\right) \\
& =\lim _{n \rightarrow \infty} E_{\mathcal{S}^{M, n}}\left(h_{n}, \Lambda_{n}\right)=E_{\mathcal{S} M}\left(f^{*}, \Gamma^{*}\right)
\end{aligned}
$$

where the first inequality uses the lower-semicontinuity of $E_{\mathcal{S}^{M}}$ (which follows from results from [24]), the second inequality follows from the optimality of $\left(f_{n}^{*}, \Gamma_{n}^{*}\right)$, and the equalities follow from the continuity of the first two terms of $E_{\mathcal{S}}$ with repect to dilations of the boundary (see [24]). Therefore, $E_{\mathcal{S} M}(f, \Gamma)=E_{\mathcal{S} M}\left(f^{*}, \Gamma^{*}\right)$ so that $(f, \Gamma)$ minimizes $E_{\mathcal{S}^{M}}$.

\section{Discussion and Summary}

We presented two procedures for partially discretizing the variational formulation of the segmentation problem for which we proved convergence results in the continuum limit. This problem was originally considered in $[14,15]$ where a method like that of Section 3 (but using Minkowski content for the length term) was shown to have similar convergence properties. There are several reasons for considering other methods such as those presented here. First, the proofs of the convergence results for the techniques presented here are much simpler (using some powerful results on Hausdorff measure) than that of $[14,15]$. Second, certain discretization methods and cost terms may be easier to implement and computationally more advantageous than others. Finally, considering several alternative approaches might suggest certain general properties that are shared by all discretizations of the original problem.

Regarding the second and third points we have some specific ideas in mind. Both approaches presented here as well as the method using Minkowski content are computationally unattractive compared to the standard discretization. For example, in the standard discretization the cost term associated with a discrete boundary is obtained by simply taking the total length of the segments in the discrete boundary. On the other hand, with the digitizations proposed, the computation of the cost term of Section 3 or of Minkowski content is much more involved. In particular, the MRF corresponding to the standard discretization is very simple, requiring no interactions between the boundary sites, while implementing the discretizations proposed would require large neighborhood structures (growing unboundedly as the lattice spacing tends to zero) and complex potentials.

The distinction can also be formalized along the following lines. Consider a distributed implementation in which there is a processor at each lattice site. The state of a processor is either zero or one depending on whether or not the boundary passes through the associated lattice square. To compute the length terms of Section 2 or using Minkowski content, each processor must perform a computation depending on the state of a very large number (tending to infinity) of other processors 
as the lattice spacing tends to zero. On the other hand, for the cost term of the usual discretization, the contribution of a particular processor to the total cost depends on the state of the processor but is independent of the state of all other processors (regardless of the discretization level). Hence, if implemented in parallel architectures in the natural way, the two methods that possess the proper convergence properties require computations that are in some sense nonlocal as the lattice spacing tends to zero, while the usual discretization results in a local computation (independent of the discretization level) but fails to have the right convergence properties. Note that for the method using piecewise linear approximations, if implemented in the natural way, the computation can be done locally but each processor requires an unbounded number of states in the continuum limit (to indicate whether an endpoint of the line segment is present at that processor and, if so, at which processor the other endpoint lies).

A natural question is whether the computational difficulties discussed above can be circumvented by a clever discrete approximation. We have obtained some results suggesting that for rectangular lattices the difficulties are not merely due to a poor choice of discrete approximations, but are inherent difficulties associated with any discrete approximation to measures of length. This result probably holds for many other regular lattices as well (e.g., hexagonal). However, interestingly the problems with nonlocal computation can be avoided for certain random tesselations (and probably for appropriate deterministic ones as well).

Also, as alluded to above, the nonlocal computations can likely be avoided if the processors are allowed to have infinitely many states. For example, this could correspond to associating a direction (or local tangent) to each boundary element in addition to just it presence or absence. Hence, in the MRF formulations this might correspond to coupled intensity and boundary fields both of which are real valued. A somewhat different approach to having real valued boundary elements is suggested by an important result of Ambrosio [1,2]. He obtained an interesting $\Gamma$-convergent approximation to the original variational problem. Specifically, he showed that the functional

$$
E^{h}(f, v)=c_{1} \iint_{\Omega}(f-g)^{2}+c_{2} \iint_{\Omega}\left(1-v^{2}\right)^{h}\|\nabla f\|^{2}+c_{3}\left(\iint_{\Omega}\left(1-v^{2}\right)^{h}\|\nabla v\|^{2}+\frac{h^{2} v^{2}}{16}\right)
$$

$\Gamma$-converges to (1) as $h \rightarrow \infty$, so that minimizers of $E^{h}(f, v)$ converge to a minimizer of $E(f, \Gamma)$ as $h \rightarrow \infty$. Here $f$ is as before and $v: \Omega \rightarrow[0,1]$ plays the role of the boundaries. For finite $h, v$ represents a sort of smoothed version of $\Gamma$ in the sense of having a value close to 1 near $\Gamma$ and having a value of 0 away from $\Gamma$, and varying continuously in between. This result suggests a natural digitization of (1) by taking a finite difference approximation to $E^{h}(f, v)$ as discussed in [25] and [11]. However, as far as we know, a proof of convergence for such finite difference approximations is lacking in this case. We expect that convergence should hold as long as $h \rightarrow \infty$ appropriately as the lattice spacing $1 / n \rightarrow 0$, namely $h / n \rightarrow 0$. Such a conjecture is natural in light of the results of $[14,15]$ and was in fact stated in [25]. Furthermore, convergence issues aside, it is not clear that computational difficulties are avoided with these approaches. E.g., in Ambrosio's approximation, there may be some computational or numerical problems as $h \rightarrow \infty$. Further work needs to be done to understand whether any computational difficulties arise in this case. 


\section{Acknowledgments}

We would like to thank Jack Koplowitz for suggesting the use of the alternate cost term of Section 3, and Eugene Wong for suggesting the piecewise linear approximation of Section 4 .

\section{References}

[1] Ambrosio, L., " Existence Theory for a New Class of Variational Problems," Center for Intelligent Control Systems Report CICS-P-93, M.I.T., 1988.

[2] Ambrosio, L., " Variational Problems in SBV," Center for Intelligent Control Systems Report CICS-P-86, M.I.T., 1988.

[3] Attouch, H., Variational Convergence for Functions and Operators, Pitman Publishing Inc., 1984.

[4] Blake, A. and A. Zisserman, "Invariant surface reconstruction using weak continuity constraints," Proc. IEEE Conf. Computer Vision and Pattern Recognition, Miami, pp. 62-67, 1986 .

[5] Blake, A. and A. Zisserman, Visual Reconstruction MIT Press, 1987.

[6] De Giorgi, E., "Г-convergenza e G-convergenza," Boll. Un. Mat. Ital., (5) 14-A, pp. 213-220, 1977.

[7] De Giorgi, E., "Convergence problems for functionals and operators," Proc. Int. Meeting on Recent Methods in Nonlinear Analysis, Rene 1978, ed. E. De Giorgi, Magenes, Mosco Pitagora, Bologna, pp. 131-188, 1979.

[8] De Giorgi, E., "New problems in $\Gamma$-convergence and G-convergence," Proc. Meeting on Free Boundary Problems, Pavia 1979, Istituto Nazionale di Atta Matematica, Roma, Vol. II, pp. 183-194, 1980.

[9] Falconer, K.J., The Geometry of Fractal Sets, Cambridge University Press, 1985.

[10] Federer, H., Geometric Measure Theory, Springer-Verlag, 1969.

[11] Geiger, D. and A. Yuille, "A common framework for image segmentation by energy functions and nonlinear diffusion," A.I. Lab Memo, M.I.T., 1989.

[12] Geman, S. and D. Geman, "Stochastic Relaxation, Gibbs Distributions, and the Bayesian Restoration of Images," IEEE Trans. Pattern Analysis and Machine Intelligence 6, pp. 721$741,1984$.

[13] Koplowitz, J. and A. Bruckstein, "Design of perimeter estimators for digitized planar shapes," SPIE Vol. 1001 Visual Communications and Image Processing, pp. 756-763, 1988.

[14] Kulkarni, S.R., "Minkowski content and lattice approximations for a variational problem," Center for Intelligent Control Systems Report CICS-95, M.I.T., 1988. 
[15] Kulkarni, S.R., S.K. Mitter, T.J. Richardson, "An existence result and lattice approximations for a variational problem," Signal Processing, Part I: Signal Processing Theory, IMA Vol. 22, edt. by Auslander et al., Springer-Verlag, pp. 189-210, 1990.

[16] Mandelbrot, B.B., The Fractal Geometry of Nature, W.H. Freeman and Company, 1982.

[17] Marroquin, J.L., "Probabilistic Solution of Inverse Problems," Ph.D. Thesis, Dept. of E.E.C.S., M.I.T., 1985.

[18] Marroquin, J., S. Mitter, T. Poggio, "Probabilistic solution of ill-posed problems in computational vision," J. of the Amer. Stat. Soc., Vol. 82, No. 397, pp. 77-89, 1987.

[19] Minsky, M and S. Papert, Perceptrons: An Intruduction to Computational Geometry, The MIT Press, 1969.

[20] Montanari, U., "Continuous skeletons from digitized images," Jornal of the ACM, Vol. 16, No. 4, pp.534-549, 1969.

[21] Montanari, U., "A note on minimal length polygonal approximation to a digitized contour," Communications of the ACM, Vol. 13, No. 1, 1970.

[22] Mumford, D. and J. Shah, "Boundary detection by minimizing functionals," Proc. IEEE Conf. Computer Vision and Pattern Recognition, San Francisco, pp. 22-26, 1985.

[23] Mumford, D. and J. Shah, "Optimal Approximations by Piecewise Smooth Functions and Associated Variational Problems," Center for Intelligent Control Systems Report CICS-P-68, M.I.T., 1988. Submitted to Communications on Pure and Applied Mathematics.

[24] Richardson, T.J., "Existence Result for a Problem Arising in Computer Vision," Center for Intelligent Control Systems Report CICS-P-63, M.I.T., 1988.

[25] Richardson, T.J., "Scale Independent Piecewise Smooth Segmentation of Images Via Variational Methods," Ph.D. Thesis, Dept. of E.E.C.S., M.I.T., 1989.

[26] Rogers, C.A., Hausdorff Measure, Cambridge University Press, 1970.

[27] Shah, J., "Segmentation by Minimizing Functionals: Smoothing Properties." To be published.

[28] Wang, Y., Harvard University, Unpublished Notes, 1988. 